\title{
A CHARACTERIZATION OF $M$-SPACES IN THE CLASS OF SEPARABLE SIMPLEX SPACES
}

$\mathrm{BY}$

\author{
ALAN GLEIT $\left({ }^{1}\right)$
}

ABSTRACT. We show that a separable simplex space is an $M$-space iff the arbitrary intersection of closed ideals is always an ideal.

Edward Effros in [5, end of $\$ 3]$ was unable to determine if an arbitrary intersection of closed ideals in a simplex space was necessarily an ideal. That this was not true in general was shown by J. Bunce [2] and F. Perdrizet. Here we shall show that for separable simplex spaces this property is equivalent to the simplex space being a (Kakutani) M-space.

In $\$ 1$ we extend some of the results of [7]. We show that certain subspaces of certain simplex spaces are again simplex spaces. In $\$ 2$ we give the aforementioned characterization.

I should like to thank the referee for his comments. The much simplified proof of Theorem 1.2 and its extension to the nonstandard case are due to him.

0 . Conventions. All vector spaces are assumed to have nonzero elements. The term measure will always denote a regular bounded Borel measure.

We use $\delta(q)$ for the point measure at $q$.

1. An existence theorem. An ordered Banach space $V$ with closed positive cone is a simplex space if its dual is a (Kakutani) $L$-space. If $Y$ is a compact Hausdorff space, we let $C(Y)$ be the space of (real) continuous functions on $Y$ with the natural pointwise order and the supremum norm. Obviously, $C(Y)$ is a simplex space. Its dual, $C^{*}(Y)$, is the space of all measures on $Y$. More generally, if $X$ is a Borel subset of $Y$, we let $C^{*}(Y ; X)$ be the space of all measures on $Y$ whose total variation on $X$ is zero. Then $C^{*}(Y ; X)$ is an $L$-space and the extreme points of the positive part of its unit ball are $\{\delta(y) \mid y \in Y-X\} \cup$ $\{0\}$ [7, Proposition 1.1].

If $V$ is a simplex space, we let

Received by the editors August 12, 1970 and, in revised form, August 28, 1971.

AMS 1969 subject classifications. Primary 4606; Secondary 4610, 4620, 4625.

Key words and phrases. M-space, simplex space, order ideals.

(1) An NSF Postdoctoral Fellow supported by NSF development grant GU 2056. 


$$
P_{1}(V)=\left\{f \in V^{*} \mid\|f\| \leq 1, f(x) \geq 0 \text { for each } x \geq 0\right\}
$$

and $E P_{1}(V)$ be its extreme points. We take

$$
E P_{1}(V)^{+}=E P_{1}(V)-\{0\}, \quad Z(V)=\text { weak }^{*} \text { closure of } E P_{1}(V)^{+} \text {. }
$$

Since $P_{1}(V)$ is a simplex, each $q \in P_{1}(V)$ is the resultant of a unique maximal probability measure $\mu_{q}$. We take $\pi_{q}=\mu_{q}-\mu_{q}(\{0\}) \delta(0)$. If $V$ is separable, $\mu_{q}$ is supported by $E P_{1}(V)$ and $\pi_{q}$ by $E P_{1}(V)^{+}$.

We may characterize $V$ as the set of affine continuous functions on $Z(V)$ vanishing at zero. Hence

$$
V=\left\{f \in C(Z(V)) \mid f(q)=\pi_{q}(f) \text { for each } q \in Z(V)-E P_{1}(V)^{+}\right\} .
$$

Let

$$
X(V)=\left\{\delta(q)-\pi_{q} \mid q \in Z(V)-E P_{1}(V)^{+}\right\} .
$$

We shall always assume that $X(V)$ is given the weak* topology relative to $C(Z(V))$.

We will be using the following well-known results repeatedly and include them for completeness. First, a map $x \rightarrow \eta_{x}$ of a topological space $E$ into the measures on a locally compact space $F$ is weak* Borel measurable iff $x \rightarrow$ $\eta_{x}(f)$ is a Borel measurable function for each $f \in C(F)$. It is bounded if sup $\left\|\eta_{x}\right\|<\infty$. We then have $[1, \mathrm{~V}, \S 3$, Proposition 2 , Definition 3 , Corollary to Proposition 12]

Lemma 1.1. Let $E$ and $F$ be locally compact Hausdorff spaces. Suppose $x \rightarrow \eta_{x}$ is a weak* bounded Borel measurable map of $E$ into the positive measures on $F$. Let $\mu$ be a positive measure on $E$.

1. Then there is a measure $\nu$ on $E$ defined by

$$
\nu=\int \eta_{x} d \mu(x)
$$

2. Suppose $f$ is a bounded universally measurable function on $F$. Then

$$
x \rightarrow \int f(y) d \eta_{x}(y)
$$

is universally measurable and

$$
\int f(y) d \nu=\int d \mu(x) \int f(y) d \eta_{x}(y)
$$

Our first result identifies $V^{\perp}$ for $V$ a simplex space. Throughout, we always consider $V$ as a subset of $C(Z(V))$ and not as a subset of $C\left(P_{1}(V)\right)$.

Theorem 1.2. Let $V$ be a simplex space. Let $Z=Z(V)$. Then $V^{\perp} \subseteq C^{*}(Z)$ may be identified as follows: 


$$
\begin{aligned}
& V^{+}=\left\{w \in C^{*}(Z) \mid \text { there is a measure } \nu \text { on } Z\right. \text { such that } \\
& \left.\qquad w(f)=\int_{Z}\left(\delta(z)-\pi_{z}\right)(f) d \nu \text { for each } f \in C(Z)\right\} .
\end{aligned}
$$

Proof. Let $w \in V^{\perp}$. We may assume that $\|w\| \leq 1$. Writing $w=w^{+}-w^{-}$, we obviously have $\left\|w^{+}\right\| \leq 1,\left\|w^{-}\right\| \leq 1$. Let

$$
w_{1}=w^{+}+\left(1-\left\|w^{+}\right\|\right) \delta(0), \quad w_{2}=w^{-}+\left(1-\left\|w^{-}\right\|\right) \delta(0) .
$$

Then $w_{1}, w_{2}$ are probability measures on $P_{1}(V)$. Let $b$ be a continuous affine function on $P_{1}(V)$. Then $b=f+b(0) 1$ for some $f \in V$. Since $w^{ \pm}(1)=\left\|w^{ \pm}\right\|$and $w^{+}(f)-w^{-}(f)=w(f)=0$ for $f \in V$, we have that $w_{1}(b)=w_{2}(b)$. Hence, $w_{1}$ and $w_{2}$ have the same resultant. Let $n$ be the unique maximal measure dominating both $w_{1}$ and $w_{2}$. Then [8, Theorem 30, p. 232]

$$
n=\int_{P_{1}(V)} \mu_{q} d w_{1}(q)=\int_{P_{1}(V)} \mu_{q} d w_{2}(q) .
$$

Since $w_{1}, w_{2}$ are supported by $Z \cup\{0\}$ and recalling that $\pi_{q}=\mu_{q}-\mu_{q}(\{0\}) \delta(0)$ we get

$$
w_{i}-n=\int_{Z}\left(\delta(q)-\pi_{q}\right) d w_{i}(q)+c_{i} \delta(0), \quad i=1,2,
$$

for suitable constants $c_{i}$. But then

$$
w=\int_{Z}\left(\delta(q)-\pi_{q}\right) d\left(w_{1}-w_{2}\right)+c \delta(0)
$$

for a suitable constant $c$. Noting that $\pi_{0}=0$, we may write $\nu=w_{1}-w_{2}+$ $c \delta(0)$ to get the required representation.

Conversely, any $w \in C^{*}(Z)$ which has the representation

$$
w=\int_{Z}\left(\delta(q)-\pi_{q}\right) d \nu
$$

for some measure $\nu$ obviously annihilates $V$.

Corollary 1.3. Let $V$ be a simplex space. Let $Z=Z(V), E=E P_{1}(V)^{+}$, and $X=X(V)$. Suppose $E$ is a universally measurable subset of $Z$. Then $V^{\text {J. }} \subseteq$ $C^{*}(Z)$ may be identified as follows:

$$
\begin{aligned}
V^{+} & =\text {linear } \operatorname{span}(\overline{\mathrm{co}}(X)) \\
& =\left\{w \in C^{*}(Z) \mid \text { there is a measure } \nu \text { on } Z\right. \text { such } \\
& \text { that } \left.w=\int_{Z-E}\left(\delta(z)-\pi_{z}\right) d \nu\right\} .
\end{aligned}
$$

Proof. For each $z \in E$, we have $\delta(z)=\pi_{z}$ and so the second representation is clear. The first follows from the second by approximating $\nu$ by atomic measures. 
A simplex $K$ is standard if it satisfies

1. The extreme points of $K$, denoted by $E(K)$, is a universally measurable subset of $K$.

2. Each maximal measure is supported by $E(K)$.

By Lemma 1.1 above, condition (2) is equivalent to

$2^{\prime}$. The maximal measures representing each point in the closure of $E(K)$ are supported by $E(K)$.

We call a simplex space $V$ a standard simplex space if $P_{1}(V)$ is a standard simplex. Note that all separable simplex spaces are standard.

We are now prepared to show that certain subspaces of standard simplex spaces are again simplex spaces.

Theorem 1.4. Let $V$ be a standard simplex space. Let $Z=Z(V)$ and $E=E P_{1}(V)^{+}$. Let $q_{0} \in Z-E$. Suppose $Y$ is a closed set in $Z$ satisfying

1. $Y \cap(Z-E)=\left\{q_{0}\right\}$.

2. $\pi_{q_{0}}(E-Y) \neq 0$.

Then $A=\left\{f \in V \mid f(y)=\pi_{q_{0}}(f)\right.$ for each $\left.y \in Y\right\}$ is a nontrivial simplex space with the relative order and norm. Further, $A^{*}$ is isometrically order isomorpbic to $C^{*}(Z ;(Z-E) \cup Y)$ and so $E P_{1}(A)^{+}=E-Y$.

Proof. We divide the proof into several stages. Throughout, we take $\pi_{0}$ to be $\pi_{q_{0}}$. Let $a=\pi_{0}(Y)$. Then

$$
0<\pi_{0}(E-Y) \leq 1-\alpha .
$$

A. First, let us consider the space $D$ defined by

$$
D=\left\{f \in C(Z) \mid f(y)=\pi_{0}(f) \text { for each } y \in Y\right\} \text {. }
$$

Let $X(D)=\left\{\delta(Y)-\pi_{0} \mid y \in Y\right\}$. As $y \rightarrow \delta(y)$ is continuous and $Y$ is compact, $Y$ is homeomorphic to $X(D)$. Let $m$ be a measure on $X(D)$. Then there is a measure $\lambda$ on $Y$ induced by $m$. So, for $f \in C(Z)$,

$$
\int_{X(D)} x(f) d m(x)=\int f(y) d \lambda-\pi_{0}(f) \lambda(Y) \text {. }
$$

Let

$$
\begin{aligned}
F=\left\{w \in C^{*}(Z) \mid\right. & \text { there exists a measure } \lambda \text { on } Y \text { such that } \\
& \left.w(f)=\int f(y) d \lambda-\pi_{0}(f) \lambda(Y) \text { for each } f \in C(Z)\right\} .
\end{aligned}
$$

Approximating $\lambda$ by atomic measures clearly yields

$$
F=\text { linear span }(\overline{c o}(X(D))) \text {. }
$$

Clearly, the weak* closure of $F$ is $D^{\lrcorner}$. To show that $F$ is weak* closed it suffices to show it is norm closed [3, V, 5.9]. 
In order to show $F$ is norm closed, we consider a $w \in F$. Then there is a measure $\lambda$ on $Y$ such that

$$
w(f)=\int f(y) d \lambda-\pi_{0}(f) \lambda(Y)
$$

for each $f \in C(Z)$. By Lemma 1.1, for each Borel set $B \subseteq Z$,

$$
w(B)=\lambda(B \cap Y)-\pi_{0}(B) \lambda(Y) .
$$

For each Borel set $A \subseteq Y$ we easily get

$$
\lambda(A)=w(A)+\pi_{0}(A) w(Y) /(1-\alpha)
$$

and so $w$ uniquely determines $\lambda$. Further

$$
|\lambda|(Y) \leq|w|(Y)+\pi_{0}(Y)|w(Y)| /(1-\alpha)
$$

and so

$$
\|\lambda\|=|\lambda|(Y) \leq\|w\|(1+\alpha /(1-\alpha)) .
$$

It should now be clear that $F$ is norm closed. Hence $F=D^{\perp}$.

B. The determination of $A^{2}$. It is clear that $A=D \cap V$ and so

$$
A^{\perp}=\text { weak }{ }^{*} \text { closure }\left(D^{\perp}+V^{\perp}\right) \text {. }
$$

We claim that $D^{+}+V^{\perp}$ is already weak* closed. From the representations for $D^{\perp}$ in $A$ and $V^{\perp}$ in Corollary 1.3 ,

$$
D^{\perp}+V^{\perp}=\text { linear span }\left(\operatorname{co}^{-}(X(V) \cup X(D))\right)
$$

and so again we need only demonstrate that $D^{+}+V^{+}$is norm closed. We let

$$
\begin{aligned}
W=\left\{w \in C^{*}(Z) \mid\right. & \text { there exists a measure } \nu \text { on } Z \text { and a } \\
& \text { measure } \lambda \text { on } Y \text { such that, for each } f \in C(Z), \\
& \left.w(f)=\int_{Z-E}\left(\delta(z)-\pi_{z}\right)(f) d \nu+\int f(y) d \lambda-\pi_{0}(f) \lambda(Y)\right\} .
\end{aligned}
$$

Then, from the representations for $D^{\perp}$ in $A$ and $V^{\perp}$ in Corollary 1.3, $W=$ $D^{+}+V^{\perp}$. Let $w \in W$ be determined by measures $\nu$ on $Z$ and $\lambda$ on $Y$. Then, using Lemma 1.1, for each Borel set $B \subseteq Z$,

$$
w(B)=\nu(B \cap(Z-E))-\int_{Z-E} \pi_{z}(B) d \nu+\lambda(B \cap Y)-\pi_{0}(B) \lambda(Y) .
$$

In particular, for Borel $A \subseteq Z-E-\left\{q_{0}\right\}$,

$$
\nu(A)=w(A)
$$

Also, $\nu\left(\left\{q_{0}\right\}\right)+\lambda\left(\left\{q_{0}\right\}\right)=w\left(\left\{q_{0}\right\}\right)$. We may, by transferring an atom if necessary, take $\lambda\left(\left\{q_{0}\right\}\right)=0$. Hence

$$
\nu\left(\left\{q_{0}\right\}\right)=w\left(\left\{q_{0}\right\}\right), \quad \lambda\left(\left\{q_{0}\right\}\right)=0 .
$$

Finally, for Borel $C \subseteq Y-\left\{q_{0}\right\}$, 


$$
\lambda(C)=w(C)+\int_{Z-E} \pi_{z}(C) d \nu+\pi_{0}(C) \lambda(Y)
$$

with $\lambda(Y)$ found by consistency. With the choice $(1.4)^{\prime \prime \prime}, \nu|(Z-E)=w|(Z-E)$ and so we may rewrite $W$ as

$$
\begin{aligned}
& W=\left\{w \in C^{*}(Z) \mid \text { there exists a measure } \lambda \text { on } Y\right. \text { without } \\
& \text { an atom at } q_{0} \text { such that, for each } f \in C(Z) \text {, } \\
& \left.w(f)=\int_{Z-E}\left(\delta(z)-\pi_{z}\right)(f) d w+\int f(y) d \lambda-\pi_{0}(f) \lambda(Y)\right\} .
\end{aligned}
$$

In this representation, we note that if $w \in C^{*}(Z)$ is determined by the measure $\lambda$ on $Y$, then again

$$
\|\lambda\| \leq\|w\|(1+\alpha /(1-\alpha)) .
$$

So clearly $W$ is norm closed. Therefore $W=A^{\perp}$.

C. The determination of $A^{*}$. The dual of $A$ is $C^{*}(Z) / A^{\perp}$. To complete the proof we need only show that $C^{*}(Z) / A^{\perp}$ is isometric order isomorphic to $C^{*}(Z ;(Z-E) \cup Y)$. We claim that each class of $A^{*}$ contains one and only one member of $C^{*}(Z ;(Z-E) \cup Y)$. Indeed, let $m \in A^{*}$ and suppose $n_{1}, n_{2} \in m$ each were in $C^{*}(Z ;(Z-E) \cup Y)$. Then $n_{1}-n_{2}=w \in W \cap C^{*}(Z ;(Z-E) \cup Y)$. Hence, there is a measure $\lambda$ on $Y$ such that for each Borel $B \subseteq Z$ (using (1.4) ${ }^{\prime}$ and the representation (1.5) for $W$ )

$$
w(B)=w(B \cap(Z-E))-\int_{Z-E} \pi_{\boldsymbol{z}}(B) d w+\lambda(B \cap Y)-\pi_{0}(B) \lambda(Y) .
$$

If $w$ vanishes on $Z-E$, then $w(B)=\lambda(B \cap Y)-\pi_{0}(B) \lambda(Y)$. Using $w(Y)=0$ we get $\lambda(Y)=0$. But then, since $w$ vanishes on $Y, \lambda=0$ and so $w \equiv 0$. Thus $n_{1}=n_{2}$.

On the other hand, let $n \in m$. We define a measure $\lambda(n)$ on $Y$ by

$$
\begin{aligned}
\lambda(n)\left(\left\{q_{0}\right\}\right) & =0, \\
\lambda(n)(A) & =\int_{Z-E} \pi_{z}(A) d n+n(A)+\pi_{0}(A) \lambda(n)(Y)
\end{aligned}
$$

for each Borel $A \subseteq Y-\left\{q_{0}\right\}$, where $\lambda(n)(Y)$ is found by consistency. Let $w(n)$ be the element of $W$ determined by $n$ and $\lambda(n)$ by (1.3). Then $n-w(n) \in m$. Using $(1.4)^{\prime \prime}$ to $(1.4)^{\prime \prime \prime \prime}$ and (1.7) one easily verifies that $w(n)(B)=n(B)$ for each Borel set $B \subseteq(Z-E) \cup Y$. Hence, $n-w(n) \in C^{*}(Z ;(Z-E) \cup Y)$ and the claim is established.

The element $n-w(n)$ depends only on the class $m$ and not on the particular representative $n$. We may therefore define a map $\phi: A^{*} \rightarrow C^{*}(Z ;(Z-E) \cup Y)$ by $\phi(m)=n-w(n)$ for each $m \in A^{*}$ and any representative $n \in m$. Obviously, $\phi$ is a linear, one-to-one map of $A^{*}$ onto $C^{*}(Z ;(Z-E) \cup Y)$. It is positive. 
Indeed, let $m$ be positive and so $n$ is positive. We must show that $(n-w(n))(B)$ $\geq 0$ for each Borel $B \subseteq Z$. This is trivial for $B \subseteq(Z-E) \cup Y$ so assume $B \subseteq$ $E-Y$. But then $(1.4)^{\prime}$ yields

$$
w(n)(B)=-\int_{Z-E} \pi_{Z}(B) d n-\pi_{0}(B) \lambda(n)(Y)
$$

and so $n-w(n)$ is indeed positive.

Last, we need show that $\phi$ is an isometry to complete the proof. Let $n \in m \in A^{*}$. Then

$$
\|\phi(m)\|=|\phi(m)|(E-Y)=|n-w(n)|(E-Y) \leq|n|(E-Y)+|w(n)|(E-Y) .
$$

For any Borel $B \subseteq E-Y$, using (1.7) and (1.8), we get

$$
\begin{aligned}
w(n)(B) & =-\int_{Z-E} \pi_{z}(B) d n-\pi_{0}(B)\left(\int_{Z-E} \pi_{z}(Y) d n+n(Y)\right) /(1-\alpha) \\
& =-\int_{Z-E}\left(\pi_{z}(B)+\pi_{z}(Y) \frac{\pi_{0}(B)}{1-\alpha}\right) d n-n(Y) \frac{\pi_{0}(B)}{1-\alpha} .
\end{aligned}
$$

Then

$$
\begin{aligned}
|w(n)|(E-Y) & \leq \int_{Z-E}\left(\pi_{z}(E-Y)+\pi_{z}(Y) \frac{\pi_{0}(E-Y)}{1-\alpha}\right) d|n|+|n|(Y) \frac{\pi_{0}(E-Y)}{1-\alpha} \\
& \leq \int_{Z-E}\left(\pi_{z}(E-Y)+\pi_{z}(Y)\right) d|n|+|n|(Y) \text { by }(1.1) \\
& \leq|n|(Z-E)+|n|(Y) .
\end{aligned}
$$

Thus, we finally get $\|\phi(m)\| \leq|n|(E-Y)+|n|(Z-E)+|n|(Y)=|n|(Z)=\|n\|$. Since $\|m\|=$ inf $_{n \in m}\|n\|$, we have $\|m\|=\|\phi(m)\|$.

The same proof also establishes the following [cf. 7, Theorem 1.2].

Corollary 1.5. Let $V, Z, E$ be as in Theorem 1.4. Let $X$ be a closed proper subset of $Z-E$. Let $x \rightarrow \mu_{x}$ be a weak* continuous map of $X$ into $P_{1}(C(Z))$. Let $X=X_{1} \cup X_{2}$ where $X_{2}=\left\{x \in X \mid \mu_{x}=\delta(x)\right\}$. We assume

1. For each $x \in X \cap(Z-E), \mu_{x}=\pi_{x}$.

2. For each $x \in X_{1}, \mu_{x}\left(X_{1} \cup(Z-E)\right)=0$.

3. $X_{1} \cup(Z-E) \neq Z$.

Then $A=\left\{f \in V \mid f(x)=\mu_{x}(f)\right.$ for each $\left.x \in X\right\}$ is a nontrivial simplex space with the relative norm and order. Further, $A^{*}$ is isometrically order isomorphic to $C^{*}\left(Z ; X_{1} \cup(Z-E)\right)$.

2. The characterization. A subset $F$ of a convex compact set $K$ is called a face if it is convex and satisfies the following condition: if $a x+(1-\alpha) y \in F$ with $x, y \in K$ and $0<\alpha<1$, then $x, y \in F$. The following extension theorem is a well-known consequence of the Edwards separation theorem [4].

Lemma 2.1. Let $F$ be a closed face of a simplex $K$. Suppose $f_{1}, f_{2}$ are continuous 
affine functions on $K$. Let $\bar{g}$ be a continuous affine function on $F$ satisfying $\alpha \geq \bar{g} \geq f_{1}, f_{2}$ for some $\alpha \in \mathbf{R}$. Then there exists a continuous affine extension $g$ of $g$ to $K$ which satisfies $a \geq g \geq f_{1}, f_{2}$.

Lemma 2.2. Let $V$ be a simplex space. Let $q \in Z(V)-E P_{1}(V)$. Suppose there exists $p \in \operatorname{supp} \pi_{q} \cap E P_{1}(V)^{+}$and a net $\left\{x_{\beta}\right\} \subseteq E P_{1}(V)^{+}-\{p\}$ which converges weak* to q. Suppose, further, there is an element $f \in V$ such that

1. $x_{\beta}(f)=0=q(f)$ for all $\beta$.

2. $p(f)>0$.

Then there exists a collection of closed maximal ideals $I_{\beta}$ such that $\cap I_{\beta}$ is not an ideal. If the net is a sequence, then the collection of ideals is countable.

Proof. Let $F_{\beta}=\left\{a x_{\beta} \mid 0 \leq a \leq 1\right\}$. Then $F_{\beta}$ is a maximal face containing zero of $P_{1}(V)$. Let $I_{\beta}$ be the annihilator of $F_{\beta}$ within $V$. Then $I_{\beta}$ is a closed maximal ideal [5, Corollary 3.2]. Since $x_{\beta}(f)=0, f \in I_{\beta}$ and so $f \in \cap I_{\beta}$.

Suppose $\cap I_{\beta}$ is an ideal. Then there would be a $v \in V^{+}, v \in \cap I_{\beta}$, and $v \geq f$. Thus, $v \in I_{\beta}$ for each $\beta$ and so $x_{\beta}(v)=0$. Therefore $q(v)=\lim x_{\beta}(v)=0$. Since $v \in V^{+}$, it is zero on the smallest closed face containing $q$ and so $p(v)=0$. However, this contradicts the assumptions that $p(f)>0$ and $v \geq f$.

We are now prepared for our characterization.

Theorem 2.3. Let $V$ be a separable simplex space. Then the following are equivalent:

1. $V$ is an M-space.

2. The intersection of an arbitrary collection of closed (maximal) ideals is always an ideal.

3. The intersection of a countable collection of closed (maximal) ideals is always an ideal.

Proof. (1) $\Rightarrow(2) \Rightarrow(3)$ are obvious.

Not $(1) \Rightarrow$ not (3). Assume $V$ is not an $M$-space. Let $Z=Z(V)$ and $E=$ $E P_{1}(V)^{+}$. Then there is an element $q \in Z-E$ such that supp $\pi_{q}$ has at least two points ([10, Theorem 2], [6, Corollary 2.6]). So choose distinct points $p_{1}$, $p_{2} \in E \cap \operatorname{supp} \pi_{q}$. Since $q$ is in the closure of $E$, there is a sequence $\left\{x_{n}\right\} \subseteq$ $E-\left\{p_{1}, p_{2}\right\}$ such that $\lim x_{n}=q$.

Let $A$ be defined by $A=\left\{v \in V \mid x_{n}(v)=q(v)=\pi_{q}(v), n=1,2, \cdots\right\}$. Then $A$ is a nontrivial simplex space with $E P_{1}(A)^{+}=E-\left\{x_{n} \mid n=1,2, \cdots\right\}$ by Theorem 1.4. Hence, $p_{1}$ and $p_{2}$ are in $E P_{1}(A)^{+}$. Let $F=\left\{\alpha p_{1}+\beta p_{2} \mid 0 \leq \alpha\right.$, $0 \leq \beta, \alpha+\beta \leq 1\}$. Then $F$ is a closed face of the simplex $P_{2}(A)$. On $F$, define continuous affine functions $\bar{g}_{1}$ and $\bar{g}_{2}$ by

$$
\bar{g}_{1}\left(\alpha p_{1}+\beta p_{2}\right)=\alpha, \quad g_{2}\left(\alpha p_{1}+\beta p_{2}\right)=\beta \text {. }
$$


Then $\bar{g}_{i} \geq 0, i=1,2$, on $F$ and so there exist elements $g_{1}, g_{2} \in A$ such that $g_{i} \geq 0$ and $g_{i} \mid F=\bar{g}_{i}$ by Lemma 2.1. Since $p_{1}\left(g_{1}\right)=1$ and $p_{2}\left(g_{2}\right)=1$, we must have $q\left(g_{1}\right)>0$ and $q\left(g_{2}\right)>0$. Let

$$
f=g_{1}-\left(q\left(g_{1}\right) / q\left(g_{2}\right)\right) g_{2} .
$$

Then obviously $f \in A \subseteq V, q(f)=0$ and $p_{1}(f)>0$. Since $f \in A$, we also have $x_{n}(f)=0, n=1,2, \cdots$. Hence, Lemma 2.2 implies that (3) is not true.

We note that Theorem 1.4 and Lemma 2.2 allow us to conclude more than just Theorem 2.3. Let $V$ be a standard simplex space and suppose $q \in Z(V)$ $E P_{1}(V)^{+}$does not lie in the rays of $P_{1}(V)$. Suppose we could find a net $\left\{x_{\beta}\right\} \subseteq$ $E P_{1}(V)^{+}$converging to $q$ such that $Y=\left\{x_{\beta}\right\}^{-}$satisfies the hypotheses of Theorem 1.4. We could then conclude the existence of a collection of closed ideals whose intersection is not an ideal.

\section{REFERENCES}

1. N. Bourbaki, Livre V: Intégration. Chap. V: Intégration des mesures, 2 ième éd., Actualités Sci. Indust., no. 1244, Hermann, Paris, 1967. MR 35 \#322.

2. J. Bunce, The intersection of closed ideals in a simplex space need not be an ideal, J. London Math. Soc. (2) 1 (1969), 67-68. MR 39 \#6050.

3. N. Dunford and J. T. Schwartz, Linear operators. I: General theory, Pure and Appl. Math., vol. 7, Interscience, New York, 1958. MR $22 \sharp 8302$.

4. D. A. Edwards, Séparation des fonctions réelles définies sur un simplexe de Choquet, C. R. Acad. Sci. Paris 261 (1965), 2798-2800. MR $32 \sharp 8131$.

5. E. Effros, Structure in simplexes, Acta Math. 117 (1967), 103-121. MR 34 \#3287.

6. E. G. Effros and A. Gleit, Structure in simplexes. III. Composition series, Trans. Amer. Math. Soc. 142 (1969), 355-379. MR $40 \# 687$.

7. A. Gleit, On the existence of simplex spaces, Israel J. Math. 9 (1971), 199-209.

8. P. A. Meyer, Probability and potentials, English ed., Blaisdell, Waltham, Mass., 1966. MR $34 \# 5119$.

9. R. Phelps, Lectures on Choquet's theorem, Van Nostrand, Princeton, N. J., 1966. MR $33 \# 1690$.

10. M. Rogalski, Espaces de Banach ordonnés, simplexes, frontières de Silov et probleme de Dirichlet, C. R. Acad. Sci. Paris Sér. A-B 263 (1966), A726-A729. MR $34 \sharp 4884$.

\section{DEPARTMENT OF MATHEMATICS, CARNEGIE-MELLON UNIVERSITY, PITTSBURGH, PENNSYLVANIA 15213}

Current address: Department of Mathematics, University of Massachusetts, Amherst, Massachusetts 01002 